\title{
DENOISING USING PROJECTIONS ONTO THE EPIGRAPH SET OF CONVEX COST FUNCTIONS
}

\author{
Mohammad Tofighi, Kivanc Kose*, and A. Enis Cetin \\ Department of Electrical and Electronic Engineering, Bilkent University, Ankara, Turkey \\ *Dermatology Department, Memorial Sloan Kettering Cancer Center, New York, USA \\ tofighi@ee.bilkent.edu.tr, ${ }^{*}$ kosek@ @ mskcc.org, cetin@ bilkent.edu.tr
}

\begin{abstract}
A new denoising algorithm based on orthogonal projections onto the epigraph set of a convex cost function is presented. In this algorithm, the dimension of the minimization problem is lifted by one and feasibility sets corresponding to the cost function using the epigraph concept are defined. As the utilized cost function is a convex function in $\mathbb{R}^{N}$, the corresponding epigraph set is also a convex set in $\mathbb{R}^{N+1}$. The denoising algorithm starts with an arbitrary initial estimate in $\mathbb{R}^{N+1}$. At each step of the iterative denoising, an orthogonal projection is performed onto one of the constraint sets associated with the cost function in a sequential manner. The method provides globally optimal solutions for total-variation, $\ell_{1}, \ell_{2}$, and entropic cost functions. ${ }^{1}$
\end{abstract}

Index Terms - Epigraph of a cost function, denoising, Projection onto convex sets, total variation

\section{INTRODUCTION}

A new denoising algorithm based on orthogonal Projections onto the Epigraph Set of a Convex cost function (PESC) is introduced. In Bregman's standard POCS approach $[1,2]$, the algorithm converges to the intersection of convex constraint sets. In this article, it is shown that it is possible to use a convex cost function in a POCS based framework using the epigraph set and the new framework is used in denoising [3-7].

In standard POCS approach, the goal is simply to find a vector, which is in the intersection of convex constraint sets [2, 8-29]. In each step of the iterative algorithm an orthogonal projection is performed onto one of the convex sets. Bregman showed that successive orthogonal projections converge to a vector, which is in the intersection of all the convex sets. If the sets do not intersect, iterates oscillate between members of the sets [30,31]. Since, there is no need to compute the Bregman distance in standard POCS, it found applications in many practical problems.

In PESC approach, the dimension of the signal reconstruction or restoration problem is lifted by one and sets corresponding to a given convex cost function are defined. This approach is graphically illustrated in Fig.1. If the cost function is a convex function in $\mathbb{R}^{N}$, the corresponding epigraph set is also a convex set in $\mathbb{R}^{N+1}$. As a result, the convex minimization problem is reduced to finding the $\left[\mathbf{w}^{*}, f\left(\mathbf{w}^{*}\right)\right]$ vector of the epigraph set corresponding to the cost function as shown in Fig. 1. As in standard POCS approach, the new iterative optimization method starts with an arbitrary initial estimate in $\mathbb{R}^{N+1}$ and an orthogonal projection is performed onto one of the

${ }^{1}$ This work is supported by the Scientific and Technological Research Council of Turkey (TUBITAK), under project 113E069. constraint sets. The resulting vector is then projected onto the epigraph set. This process is continued in a sequential manner at each step of the optimization problem. This method provides globally optimal solutions for convex cost functions such as total-variation [32], filtered variation [4], $\ell_{1}$ [33], and entropic function [10]. The iteration process is shown in Fig. 1. Regardless of the initial value $\underline{\mathbf{w}}_{0}$, iterates converge to $\left[\mathbf{w}^{*}, f\left(\mathbf{w}^{*}\right)\right]$ pair as shown in Fig. 1 .

The article is organized as follows. In Section 2, the epigraph of a convex cost function is defined and the convex minimization method based on the PESC approach is introduced. In Section 3, the new denoising method is presented. The new approach does not require a regularization parameter as in other TV based methods [9, 20, 32]. In Section 4, the simulation results and some denoising examples, are presented.

\section{EPIGRAPH OF A CONVEX COST FUNCTION}

Let $f: \mathbb{R}^{N} \rightarrow \mathbb{R}$ be a convex cost function. We increase the dimension by one to define the epigraph set of $f$ in $\mathbb{R}^{N+1}$ as follows:

$$
\mathrm{C}_{f}=\left\{\underline{\mathbf{w}}=\left[\mathbf{w}^{T} y\right]^{T}: y \geq f(\mathbf{w})\right\},
$$

which is the set of $N+1$ dimensional vectors, whose $(N+1)^{s t}$ component $y$ is greater than $f(\mathbf{w})$. We use bold face letters for $N$ dimensional vectors and underlined bold face letters for $N+1$ dimensional vectors, respectively. Another set that is related with the cost function $f(\mathbf{w})$ is the level set:

$$
\mathrm{C}_{s}=\left\{\underline{\mathbf{w}}=\left[\mathbf{w}^{T} y\right]^{T}: y \leq 0, \underline{\mathbf{w}} \in \mathbb{R}^{N+1}\right\},
$$

where it is assumed that $f(\mathbf{w}) \geq 0$ for all $f(\mathbf{w}) \in \mathbb{R}$. Both $C_{f}$ and $C_{s}$ are closed and convex sets in $\mathbb{R}^{N+1}$. Other closed and convex sets describing a feature of the desired solution can be also used in this approach. Sets $C_{f}$ and $C_{s}$ are graphically illustrated in Fig. 1. An important component of the PESC approach is to perform an orthogonal projection onto the epigraph set. Let $\underline{\mathbf{w}}_{1}$ be an arbitrary vector in $\mathbb{R}^{N+1}$. The projection $\underline{\mathbf{w}}_{2}$ is determined by minimizing the distance between $\underline{\mathbf{w}}_{1}$ and $C_{f}$, i.e.,

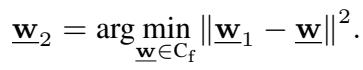

Equation (3) is the ordinary orthogonal projection operation onto the set $\mathrm{C}_{\mathrm{f}} \in \mathbb{R}^{N+1}$. In order to solve the problem in Eq. (3) we do not need to compute the Bregman's so-called D-projection or Bregman projection. Projection onto the set $C_{s}$ is trivial. We simply force the last component of the $N+1$ dimensional vector to zero. In the PESC algorithm, iterates eventually oscillate between the two nearest vectors of the sets $C_{s}$ and $C_{f}$ as shown in Fig. 1. As a result, we obtain

$$
\lim _{n \rightarrow \infty} \underline{\mathbf{w}}_{2 n}=\left[\mathbf{w}^{*} f\left(\mathbf{w}^{*}\right)\right]^{T},
$$




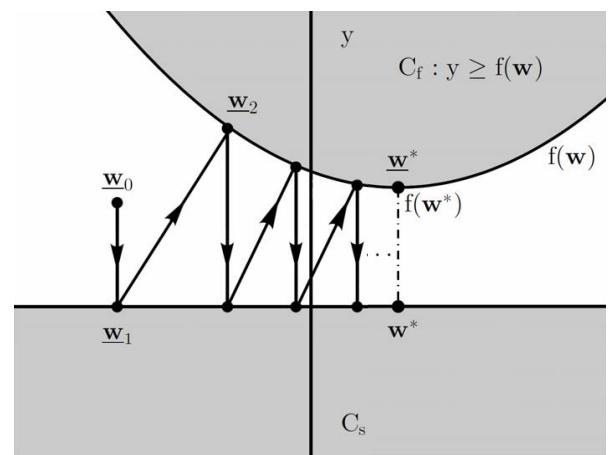

Fig. 1. Two convex sets $C_{f}$ and $C_{s}$ corresponding to the convex cost function $f$. We sequentially project an initial vector $\underline{\mathbf{w}}_{0}$ onto $C_{s}$ and $C_{f}$ to find the global minimum, which is located at $\underline{\mathbf{w}}^{*}=\left[\mathbf{w}^{*} f\left(\mathbf{w}^{*}\right)\right]^{T}$.

where $\mathbf{w}^{*}$ is the $N$ dimensional vector minimizing $f(\mathbf{w})$. The proof of Eq. (4) follows from Bregman's POCS theorem [1]. It was generalized to non-intersection case by Gubin et. al [30]. Since the two closed and convex sets $C_{s}$ and $C_{f}$ are closest to each other at the optimal solution case, iterations oscillate between the vectors $\left[\mathbf{w}^{*} f\left(\mathbf{w}^{*}\right)\right]^{T}$ and $\left[\mathbf{w}^{*} 0\right]^{T}$ in $\mathbb{R}^{N+1}$ as $n$ tends to infinity. It is possible to increase the speed of convergence by non-orthogonal projections [21].

If the cost function $f$ is not convex and have more than one local minimum then the corresponding set $C_{f}$ is not convex in $\mathbb{R}^{N+1}$. In this case iterates may converge to one of the local minima.

In current TV based denoising methods $[32,34]$ the following cost function is used:

$$
\underset{\mathbf{w}}{\operatorname{argmin}}\|\mathbf{v}-\mathbf{w}\|^{2}+\lambda \mathrm{TV}(\mathbf{w})
$$

where $\mathbf{v}$ is the observed signal. The solution of this problem can be obtained using the method in an iterative manner, by performing successive orthogonal projections onto $C_{f}$ and $C_{s}$, as discussed above. In this case the cost function is $f(\mathbf{w})=\|\mathbf{v}-\mathbf{w}\|_{2}^{2}+\lambda \mathrm{TV}(\mathbf{w})$. Therefore,

$$
C_{f}=\left\{\mathbf{w} \in \mathbb{R}^{N+1}:\|\mathbf{v}-\mathbf{w}\|^{2}+\lambda \mathrm{TV}(\mathbf{w}) \leq y\right\} .
$$

The denoising solutions that we obtained are very similar to the ones found by Chambolle's in [32] as both methods use the same cost function. One problem in [32] is the estimation of the regularization parameter $\lambda$. One has to determine the $\lambda$ in an ad-hoc manner or by visual inspection. In the next section, a new denoising method with a different TV based cost function is described. The new method does not require a regularization parameter. Concept of epigraph is first used in signal reconstruction problems in [35, 36]. We also independently developed epigraph based algorithms in [37].

\section{DENOISING USING PESC}

In this section, we present a new denoising method, based on the epigraph set of the convex cost function. It is possible to use TV, $\mathrm{FV}$ and $\ell_{1}$ norm as the convex cost function. Let the original signal or image be $\mathbf{w}_{\text {orig }}$ and its noisy version be $\mathbf{v}$. Suppose that the observation model is the additive noise model:

$$
\mathbf{v}=\mathbf{w}_{\text {orig }}+\boldsymbol{\eta},
$$

where $\boldsymbol{\eta}$ is the additive noise. In this approach we solve the following problem for denoising:

$$
\underline{\mathbf{w}}^{\star}=\underset{\underline{\mathbf{w}} \in \mathrm{C}_{\mathrm{f}}}{\arg \min }\|\underline{\mathbf{w}}\|^{2},
$$

where $\underline{\mathbf{v}}=\left[\mathbf{v}^{T} 0\right]$ and $C_{f}$ is the epigraph set of TV or FV in $\mathbb{R}^{N+1}$. The TV function, which we used for an $M \times M$ discrete image $\mathbf{w}=\left[w^{i, j}\right], \quad 0 \leq i, j \leq M-1 \in \mathbb{R}^{M \times M}$ is as follows:

$$
T V(\mathbf{w})=\sum_{i, j}\left(\left|w^{i+1, j}-w^{i, j}\right|+\left|w^{i, j+1}-w^{i, j}\right|\right) .
$$

The minimization problem (8) is essentially the orthogonal projection onto the set $C_{f}=\left\{\mathbf{w} \in \mathbb{R}^{N+1}: T V(\mathbf{w}) \leq y\right\}$. This means that we select the nearest vector $\underline{\mathbf{w}}^{\star}$ on the set $C_{f}$ to $\mathbf{v}$. This is graphically illustrated in Fig. 2. Let us explain the projection onto an epigraph set of a convex cost function $\phi$ in detail. Equation (8) is equivalent to:

$$
\underline{\mathbf{w}}^{\star}=\left[\begin{array}{c}
\mathbf{w}_{p} \\
\phi\left(\mathbf{w}_{p}\right)
\end{array}\right]=\underset{\underline{\mathbf{w}} \in \mathrm{C}_{\mathrm{f}}}{\arg \min }\left\|\left[\begin{array}{l}
\mathbf{v} \\
0
\end{array}\right]-\left[\begin{array}{c}
\mathbf{w} \\
\phi(\mathbf{w})
\end{array}\right]\right\|,
$$

where $\underline{\mathbf{w}}^{\star}=\left[\mathbf{w}_{p}^{T}, \phi\left(\mathbf{w}_{p}\right)\right]$ is the projection of $(\mathbf{v}, 0)$ onto the epigraph set. The projection $\underline{\mathbf{w}}^{\star}$ must be on the boundary of the epigraph set. Therefore, the projection must be on the form $\left[\mathbf{w}_{p}^{T}, \phi\left(\mathbf{w}_{p}\right)\right]$. Equation (10) becomes:

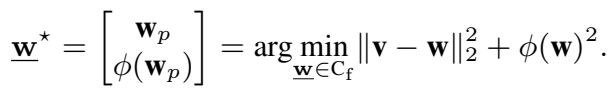

In the case of total variation $\phi(\mathbf{w})=T V(\mathbf{w})$. It is also possible to use $\lambda \phi($.$) as a the convex cost function and Eq. 11$ becomes:

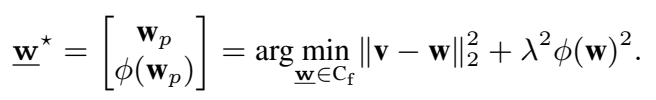

Actually, Combettes and Pesquet and other researchers including us used a similar convex set in denoising and other signal restoration applications $[4,20,34,36]$. The following convex set in $\mathbb{R}^{N}$ describes all signals whose TV is bounded by an upper bound $\epsilon$ :

$$
C_{b}=\{\mathbf{w}: \operatorname{TV}(\mathbf{w}) \leq \epsilon\} .
$$

The parameter $\epsilon$ is a fixed upper bound on the total variation of the signal and it has to be determined a priori in an ad-hoc manner. On the other hand we do not specify a prescribed number on the TV of vectors in the epigraph set approach. The upper bound on TV is automatically determined by the orthogonal projection onto $C_{f}$ from the location of the corrupted signal as shown in Fig. 2.

In current TV based denoising methods [32, 34] the following cost function is used:

$$
\min \|\mathbf{v}-\mathbf{w}\|_{2}^{2}+\lambda \mathrm{TV}(\mathbf{w}) .
$$

The solution of (14) can be obtained using the method that we discussed in Section 2. Similar to the LASSO approach [38] a major problem with this approach is the estimation of the regularization parameter $\lambda$. One has to determine the $\lambda$ in an ad-hoc manner or by visual inspection. It is experimentally observed that Eq. (12) produces good denoising results when $\lambda=1$. Experimental results are presented in Section 4. Notice that, this $C_{f}$ is different from Eq. (6). This means that we select the nearest vector $\underline{\mathbf{w}}^{\star}$ on the set $C_{f}$ to 


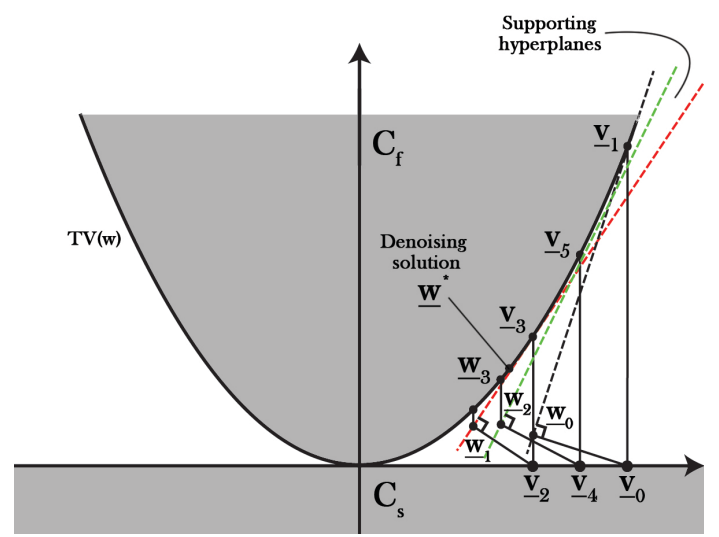

Fig. 2. Graphical representation of the minimization of Eq. (8), using projections onto the supporting hyperplanes of $C_{f}$. In this problem the sets $C_{s}$ and $C_{f}$ intersect because $T V(\mathbf{w})=0$ for $\mathbf{w}=[0,0, \ldots, 0]^{T}$ or for a constant vector.

$\underline{\mathbf{v}}_{0}$. This is graphically illustrated in Fig. 2. During this orthogonal projection operations, we do not require any parameter adjustment as in [32].

Implementation: The projection operation described in Eq. (8) can not be obtained in one step when the cost function is TV. The solution is determined by performing successive orthogonal projections onto supporting hyperplanes of the epigraph set $C_{f}$. In the first step, $\mathrm{TV}\left(\mathbf{v}_{0}\right)$ and the surface normal at $\underline{\mathbf{v}}_{1}=\left[\mathbf{v}_{0}^{T} \mathrm{TV}\left(\mathbf{v}_{0}\right)\right]$ in $\mathbb{R}^{N+1}$ are calculated. In this way, the equation of the supporting hyperplane at $\underline{\mathbf{v}}_{1}$ is obtained. The vector $\underline{\mathbf{v}}_{0}=\left[\begin{array}{ll}\mathbf{v}_{0}^{T} & 0\end{array}\right]$ is projected onto this hyperplane and $\underline{\mathbf{w}}_{0}$ is obtained as our first estimate as shown in Fig. 2. In the second step, $\underline{\mathbf{w}}_{0}$ is projected onto the set $C_{s}$ by simply making its last component zero. The TV of this vector and the surface normal, and the supporting hyperplane is calculated as in the previous step. We calculate the distance between $\underline{\mathbf{v}}_{0}$ and $\underline{\mathbf{w}}_{i}$ at each step of the iterative algorithm described in the previous paragraph. The distance $\left\|\underline{\mathbf{v}}_{0}-\underline{\mathbf{w}}_{i}\right\|^{2}$ does not always decrease for high $i$ values. This happens around the optimal denoising solution $\underline{\mathbf{w}}^{\star}$. Once we detect an increase in $\left\|\underline{\mathbf{v}}_{0}-\underline{\mathbf{w}}_{i}\right\|^{2}$, we perform a refinement step to obtain the final solution of the denoising problem. In refinement step, the supporting hyperplane at $\frac{\underline{\mathbf{v}}_{2 i+1}+\underline{\mathbf{v}}_{2 i+3}}{2}$ is used in the next iteration. For instance, when $\underline{\mathbf{v}}_{2}$ is projected, the distance is increased, therefore, in $i=0$ in Fig. 2, instead of $\underline{\mathbf{v}}_{3}$, vector $\underline{\mathbf{v}}_{5}$ will be used in next step. Next, $\underline{\mathbf{v}}_{4}$ is projected onto the new supporting hyperplane, and $\underline{\mathbf{w}}_{2}$ is obtained. In Fig. 2, by projecting the $\underline{\mathbf{w}}_{2}$ onto $C_{f}$, the vector $\underline{\mathbf{w}}_{3}$ is obtained which is very close to the denoising solution $\underline{\mathbf{w}}^{\star}$. In general iterations continue until $\left\|\underline{\mathbf{w}}_{i}-\underline{\mathbf{w}}_{i-1}\right\| \leq \epsilon$, where $\epsilon$ is a prescribed number, or iterations can be stopped after a certain number of iterations. A typical convergence graph is shown in Fig. 3 for the "note" image. It is possible to obtain a smoother version of $\underline{\mathbf{w}}^{\star}$ by simply projecting $\underline{\mathbf{v}}$ inside the set $C_{f}$ instead of the boundary of $C_{f}$.

\section{SIMULATION RESULTS}

The PESC algorithm is tested with a wide range of images. Let us start with the "Note" image shown in Fig. 6(a). This is corrupted by a zero mean Gaussian noise with $\sigma=45$ in Fig. 6(b). The image is restored using PESC, SURE-LET [39], and Chambolle's algorithm [32] and the denoised images are shown in Fig. 6(c), 6(d), and 6(e), with SNR values equal to $15.08,13.20$, and $11.02 \mathrm{~dB}$, respectively. SURE-LET and Chambolle's algorithm produce some

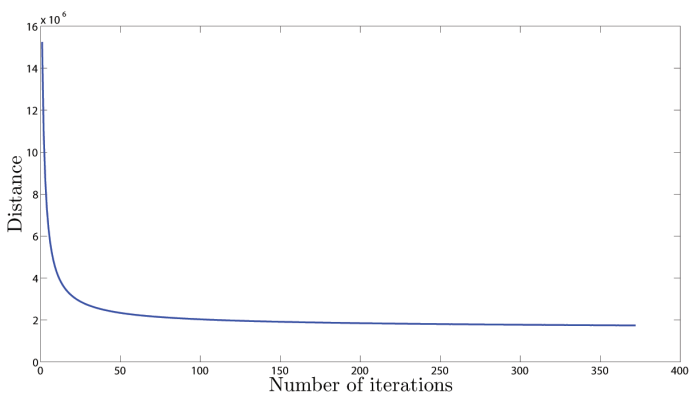

Fig. 3. Euclidian distance from $\mathbf{v}$ to the epigraph of $\mathrm{TV}$ at each iteration $\left(\left\|\underline{\mathbf{v}}-\underline{\mathbf{w}}_{i}\right\|\right)$ with noise standard deviation of $\sigma=30$.

patches of gray pixels at the background. The regularization parameter $\lambda$ in Eq. (14) is manually adjusted to get the best possible results for each image and each noise type and level in [32], and SURE-LET require the knowledge about noises standard deviation in [39]. Moreover, Structural Similarity Index (SSIM) is also calculated as in [40] for all methods. PESC algorithm not only produces higher SNR and SSIM values than other methods, but also provides visually better looking image. The same experiments are also done over "cancer cell" image, which the results are presented in Fig. 6. Denoising results for other noise levels are presented in Table 1. We also tested the PESC algorithm against $\epsilon$-contaminated Gaussian noise (salt-and-pepper noise) with the PDF of

$$
f(x)=\epsilon \phi\left(\frac{x}{\sigma_{1}}\right)+(1-\epsilon) \phi\left(\frac{x}{\sigma_{2}}\right),
$$

where $\phi(x)$ is the standard Gaussian distribution with mean zero and unit standard deviation. The results of the tests are presented in Table 3. The performance of the reconstruction is measured using the SNR criterion, which is defined as follows

$$
\mathrm{SNR}=20 \times \log _{10}\left(\frac{\left\|\mathbf{w}_{\text {orig }}\right\|}{\left\|\mathbf{w}_{\text {orig }}-\mathbf{w}_{\text {rec }}\right\|}\right),
$$

where $\mathbf{w}_{\text {orig }}$ is the original signal and $\mathbf{w}_{r e c}$ is the reconstructed signal. All the SNR values in Tables are in $\mathrm{dB}$.

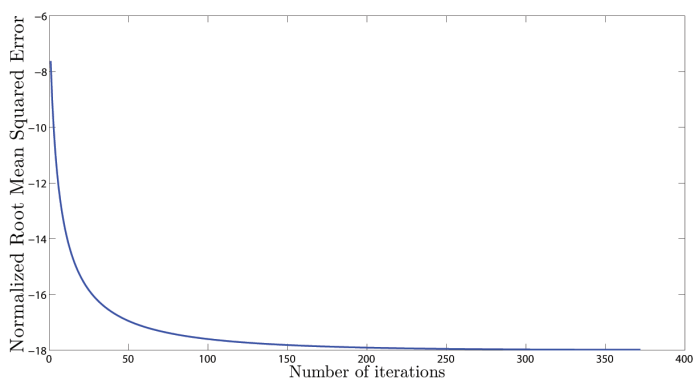

Fig. 4. NRMSE vs. iteration number for denoising the "Note" image with Gaussian noise with standard deviation of $\sigma=30$.

It is also possible to use Normalized Root Mean Square Error metric as

$$
\operatorname{NRMSE}(i)=\frac{\left\|\mathbf{w}_{i}-\mathbf{w}_{\text {orig }}\right\|}{\left\|\mathbf{w}_{\text {orig }}\right\|} \quad i=1, \ldots, N,
$$

which $N$ is the number of the iterations, in [20] to illustrate the convergence of the PESC based denoising algorithm. As shown in Fig. 4 , NRMSE value decreases as the iterations proceeds while denoising the "Note" image corrupted with Gaussian noise $(\sigma=25)$. For 


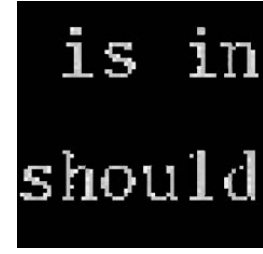

(a) Original

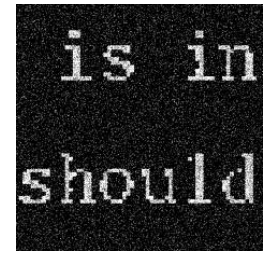

(b) Noisy

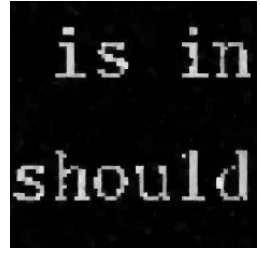

(c) PESC

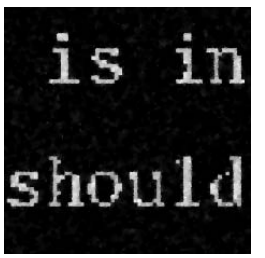

(d) Chambolle's algo.

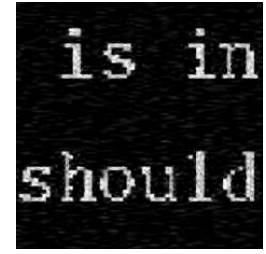

(e) SURE-LET
Fig. 5. (a) A portion of original "Note" image, (b) image corrupted with Gaussian noise with $\sigma=45$, denoised images, using: (c) PESC; SNR = $15.08 \mathrm{~dB}$ and SSIM $=0.1984$, (d) Chambolle's algorithm; SNR $=13.20 \mathrm{~dB}$ and SSIM $=0.1815$, (e) SURE-LET; SNR $=11.02 \mathrm{~dB}$ and SSIM $=0.1606$. Chambolle's algorithm and SURE-LET produce some patches of gray pixels at the background.

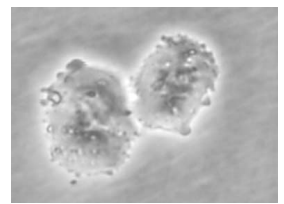

(a) Original

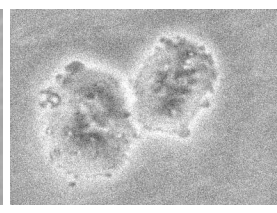

(b) Noisy

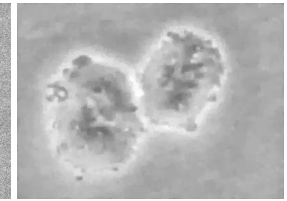

(c) PESC

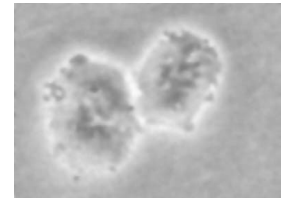

(d) Chambolle's algo.

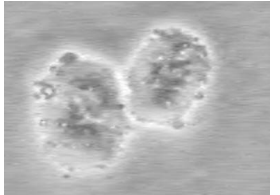

(e) SURE-LET
Fig. 6. (a) Original "Cancer cell" image, (b) image corrupted with Gaussian noise with $\sigma=20$, denoised image, using: (c) PESC; $\mathrm{SNR}=32.31 \mathrm{~dB}$ and SSIM $=0.5182$, (d) Chambolle's algorithm; $\mathrm{SNR}=31.18 \mathrm{~dB}$ and SSIM $=$ 0.3978 , (e) SURE-LET algorithm; SNR $=31.23 \mathrm{~dB}$ and SSIM $=0.4374$.

the same image another convergence metric called Normalized Total Variation, which is defined in [20] as $\operatorname{NTV}(i)=\frac{T V\left(\mathbf{w}_{i}\right)}{T V\left(\mathbf{w}_{\text {orig }}\right)}$, also converges to 1 in almost 100 iterations. In Table 2, denoising results for 34 images including 10 well-known test images from image processing literature and 24 images from Kodak Database [41], with different noise levels are presented. In almost all cases PESC method produces higher SNR and SSIM results than [32, 39].

Table 1. Comparison of the results for denoising algorithms with Gaussian noise for "note" image.

\begin{tabular}{|c|c|c|c|c|c|c|c|c|}
\hline Noise $\sigma$ & \multicolumn{2}{|c|}{ Input } & \multicolumn{2}{c|}{ PESC } & \multicolumn{2}{c|}{ Chambolle [32] } & \multicolumn{2}{c|}{ SURE-LET [39] } \\
\hline \hline & SNR & SSIM & SNR & SSIM & SNR & SSIM & SNR & SSIM \\
\hline 5 & 21.12 & 0.2201 & 30.63 & 0.2367 & 29.48 & 0.2326 & 27.42 & 0.2212 \\
\hline 10 & 15.12 & 0.2037 & 25.93 & 0.2290 & 24.89 & 0.2213 & 22.20 & 0.2086 \\
\hline 15 & 11.56 & 0.1917 & 22.91 & 0.2216 & 21.76 & 0.2141 & 19.13 & 0.1999 \\
\hline 20 & 9.06 & 0.1825 & 20.93 & 0.2165 & 19.55 & 0.2065 & 16.95 & 0.1867 \\
\hline 25 & 7.14 & 0.1716 & 19.27 & 0.2111 & 17.73 & 0.2006 & 15.34 & 0.1810 \\
\hline 30 & 5.59 & 0.1636 & 17.89 & 0.2102 & 16.43 & 0.1950 & 13.93 & 0.1767 \\
\hline 35 & 4.21 & 0.1565 & 16.68 & 0.2073 & 15.23 & 0.1903 & 12.87 & 0.1706 \\
\hline 40 & 3.07 & 0.0 .1488 & 15.90 & 0.2030 & 14.07 & 0.1855 & 11.77 & 0.1645 \\
\hline 45 & 2.05 & 0.1407 & 15.08 & 0.1984 & 13.20 & 0.1815 & 11.02 & 0.1606 \\
\hline 50 & 1.12 & 0.1332 & 14.25 & 0.1909 & 12.19 & 0.1766 & 10.17 & 0.1862 \\
\hline \hline Average & 8.00 & 0.1712 & $\mathbf{1 9 . 9 5}$ & $\mathbf{0 . 2 1 0 7}$ & 18.45 & 0.2004 & 16.08 & 0.1862 \\
\hline
\end{tabular}

Table 2. Comparison of the results for denoising algorithms under Gaussian noise with standard deviations of $\sigma$.

\begin{tabular}{|c|c|c|c|c|c|}
\hline Images & $\sigma$ & Input SNR & PESC & Chambolle [32] & SURE-LET [39] \\
\hline \hline House & 30 & 13.85 & 27.60 & 27.13 & 27.38 \\
\hline House & 50 & 9.45 & 24.61 & 24.36 & 24.59 \\
\hline Lena & 30 & 12.95 & 23.85 & 23.54 & 23.92 \\
\hline Lena & 50 & 8.50 & 21.68 & 21.37 & 21.38 \\
\hline Mandrill & 30 & 13.04 & 19.98 & 19.64 & 20.56 \\
\hline Mandrill & 50 & 8.61 & 17.94 & 17.92 & 18.22 \\
\hline Living room & 30 & 12.65 & 21.33 & 20.88 & 21.29 \\
\hline Living room & 50 & 8.20 & 19.34 & 19.05 & 19.19 \\
\hline Lake & 30 & 13.44 & 22.19 & 21.86 & 22.23 \\
\hline Lake & 50 & 8.97 & 20.26 & 19.90 & 20.07 \\
\hline Jet plane & 30 & 15.57 & 26.31 & 25.91 & 26.49 \\
\hline Jet plane & 50 & 11.33 & 24.07 & 23.54 & 24.10 \\
\hline Peppers & 30 & 12.65 & 24.24 & 23.59 & 23.78 \\
\hline Peppers & 50 & 8.20 & 22.05 & 21.36 & 21.82 \\
\hline Pirate & 30 & 12.13 & 21.43 & 21.30 & 21.27 \\
\hline Pirate & 50 & 7.71 & 19.58 & 19.43 & 19.32 \\
\hline Cameraman & 30 & 12.97 & 24.20 & 23.67 & 24.58 \\
\hline Cameraman & 50 & 8.55 & 21.80 & 21.22 & 22.06 \\
\hline Flower & 30 & 11.84 & 21.97 & 20.89 & 17.20 \\
\hline Flower & 50 & 7.42 & 19.00 & 18.88 & 13.21 \\
\hline \hline 24-Kodak(ave.) & 30 & 11.92 & 21.05 & 20.80 & 20.92 \\
\hline \hline 24-Kodak(ave.) & 50 & 7.48 & 18.97 & 18.58 & 18.88 \\
\hline \hline Average \pm std & 30 & $12.27 \pm 1.66$ & $\mathbf{2 3 . 1 2} \pm 2.35$ & $22.66 \pm 2.34$ & $22.70 \pm 2.91$ \\
\hline Average \pm std & 50 & $7.84 \pm 1.67$ & $\mathbf{2 0 . 8 5} \pm 2.17$ & $20.26 \pm 3.13$ & $20.51 \pm 2.07$ \\
\hline & & & & &
\end{tabular}

Table 3. Comparison of the results for denoising algorithms for $\epsilon$ Contaminated Gaussian noise for "note" image

\begin{tabular}{|c|c|c|c|c|c|c|}
\hline$\epsilon$ & $\sigma_{1}$ & $\sigma_{2}$ & Input SNR & PESC & Chambolle [32] & SURE-LET [39] \\
\hline \hline 0.9 & 5 & 30 & 14.64 & 23.44 & 22.26 & 16.11 \\
\hline 0.9 & 5 & 40 & 12.55 & 21.39 & 20.32 & 13.65 \\
\hline 0.9 & 5 & 50 & 10.75 & 19.49 & 18.63 & 11.64 \\
\hline 0.9 & 5 & 60 & 9.29 & 17.61 & 17.37 & 10.25 \\
\hline 0.9 & 5 & 70 & 7.98 & 16.01 & 16.24 & 8.91 \\
\hline 0.9 & 5 & 80 & 6.89 & 14.54 & 14.97 & 7.88 \\
\hline \hline 0.9 & 10 & 30 & 12.56 & 22.88 & 21.71 & 17.06 \\
\hline 0.9 & 10 & 40 & 11.13 & 21.00 & 19.97 & 14.26 \\
\hline 0.9 & 10 & 50 & 9.85 & 19.35 & 18.46 & 12.20 \\
\hline 0.9 & 10 & 60 & 8.58 & 17.87 & 17.10 & 10.69 \\
\hline 0.9 & 10 & 70 & 7.52 & 16.38 & 16.03 & 9.18 \\
\hline 0.9 & 10 & 80 & 6.46 & 15.05 & 15.12 & 8.14 \\
\hline \hline 0.95 & 5 & 30 & 16.75 & 24.52 & 23.78 & 19.12 \\
\hline 0.95 & 5 & 40 & 14.98 & 22.59 & 21.54 & 16.62 \\
\hline 0.95 & 5 & 50 & 13.41 & 20.54 & 19.91 & 14.62 \\
\hline 0.95 & 5 & 60 & 12.10 & 18.72 & 18.63 & 13.11 \\
\hline 0.95 & 5 & 70 & 10.80 & 17.13 & 17.50 & 11.71 \\
\hline 0.95 & 5 & 80 & 9.76 & 15.63 & 16.38 & 10.54 \\
\hline \hline 0.95 & 10 & 30 & 13.68 & 23.79 & 22.62 & 19.34 \\
\hline 0.95 & 10 & 40 & 12.66 & 22.09 & 21.12 & 17.06 \\
\hline 0.95 & 10 & 50 & 11.71 & 20.65 & 19.60 & 15.16 \\
\hline 0.95 & 10 & 60 & 10.72 & 19.10 & 18.30 & 13.40 \\
\hline 0.95 & 10 & 70 & 9.82 & 17.59 & 17.22 & 12.11 \\
\hline 0.95 & 10 & 80 & 8.92 & 16.12 & 16.45 & 10.91 \\
\hline & & & & & & \\
\hline
\end{tabular}

\section{CONCLUSION}

A new denoising method based on the epigraph of the TV function is developed. Epigraph sets of other convex cost functions can be also used in the new denoising approach. The denoised signal is obtained by making an orthogonal projection onto the epigraph set from the corrupted signal in $\mathbb{R}^{N+1}$. The new algorithm does not need the optimization of the regularization parameter as in standard TV denoising methods. Experimental results indicate that better SNR and SSIM results are obtained compared to standard TV based denoising in a large range of images. The proposed method can be incorporated into the so called 3-D denoising methods [42]. In 3-D denoising methods similar image blocks are grouped and shrinked according to the noise level. Since our method does not need the noise variation, it will lead to more flexible 3-D methods. 


\section{REFERENCES}

[1] L.M. Bregman, "Finding the common point of convex sets by the method of successive projection.(russian)," $\{U S S R\}$ Doklady Akademii Nauk SSSR, vol. 7, no. 3, pp. 200 - 217, 1965.

[2] D.C. Youla and H. Webb, "Image Restoration by the Method of Convex Projections: Part 1 Num2014;theory," IEEE Transactions on Medical Imaging, vol. 1, pp. 81-94, 1982.

[3] L. I. Rudin, S. Osher, and E. Fatemi, "Nonlinear total variation based noise removal algorithms," Physica D: Nonlinear Phenomena, vol. 60, pp. 259-268, 1992.

[4] K. Kose, V. Cevher, and A.E. Cetin, "Filtered variation method for denoising and sparse signal processing," IEEE ICASSP, pp. 3329-3332, 2012.

[5] O. Gunay, K. Kose, B. U. Toreyin, and A. E. Cetin, "Entropyfunctional-based online adaptive decision fusion framework with application to wildfire detection in video," IEEE Transactions on Image Processing, vol. 21, pp. 2853-2865, 2012.

[6] L.M. Bregman, "The Relaxation Method of Finding the Common Point of Convex Sets and Its Application to the Solution of Problems in Convex Programming," USSR Computational Mathematics and Mathematical Physics, vol. 7, pp. 200 - 217, 1967.

[7] W. Yin, S. Osher, D. Goldfarb, and J. Darbon, "Bregman iterative algorithms for $\ell_{1}$-minimization with applications to compressed sensing," SIAM Journal on Imaging Sciences, vol. 1, no. 1, pp. 143-168, 2008.

[8] A. E. Cetin, A. Bozkurt, O. Gunay, Y. H. Habiboglu, K. Kose, I. Onaran, R. A. Sevimli, and M. Tofighi, "Projections onto convex sets (pocs) based optimization by lifting," IEEE GlobalSIP, Austin, Texas, USA, 2013

[9] S. Ono, M. Yamagishi, and I. Yamada, "A sparse system identification by using adaptively-weighted total variation via a primal-dual splitting approach," in IEEE ICASSP, 2013, pp. 6029-6033.

[10] K. Kose, O. Gunay, and A. E. Cetin, "Compressive sensing using the modified entropy functional," Digital Signal Processing, pp. $63-70,2013$.

[11] Y. Censor, W. Chen, P. L. Combettes, R. Davidi, and G. T. Herman, "On the Effectiveness of Projection Methods for Convex Feasibility Problems with Linear Inequality Constraints," Computational Optimization and Applications, vol. 51, pp. 1065-1088, 2012.

[12] K. Slavakis, S. Theodoridis, and I. Yamada, "Online KernelBased Classification Using Adaptive Projection Algorithms," IEEE Transactions on Signal Processing, vol. 56, pp. 27812796, 2008.

[13] A. E. Cetin, "Reconstruction of signals from fourier transform samples," Signal Processing, pp. 129-148, 1989.

[14] K. Kose and A. E. Cetin, "Low-pass filtering of irregularly sampled signals using a set theoretic framework," IEEE Signal Processing Magazine, pp. 117-121, 2011.

[15] Y. Censor and A. Lent, "An Iterative Row-Action Method for Interval Convex Programming," Journal of Optimization Theory and Applications, vol. 34, pp. 321-353, 1981.

[16] S. Konstantinos, S. Theodoridis, and I. Yamada, "Adaptive constrained learning in reproducing kernel hilbert spaces: the robust beamforming case," IEEE Transactions on Signal Processing, vol. 57, pp. 4744-4764, 2009.

[17] K. S Theodoridis and I. Yamada, "Adaptive learning in a world of projections," IEEE Signal Processing Magazine, vol. 28, no. 1, pp. 97-123, 2011.

[18] Y. Censor and A. Lent, "Optimization of $\log x$ entropy over linear equality constraints," SIAM Journal on Control and Optimization, vol. 25, no. 4, pp. 921-933, 1987.

[19] H. J. Trussell and M. R. Civanlar, "The Landweber Iteration and Projection Onto Convex Set," IEEE Transactions on Acoustics, Speech and Signal Processing, vol. 33, no. 6, pp. 1632-1634, 1985.

[20] P. L. Combettes and J.-Ch. Pesquet, "Image restoration subject to a total variation constraint," IEEE Transactions on Image Processing, vol. 13, pp. 1213-1222, 2004.
[21] P. L. Combettes, "The foundations of set theoretic estimation," Proceedings of the IEEE, vol. 81, pp. 182 -208, 1993.

[22] I. Yamada, M. Yukawa, and M. Yamagishi, "Minimizing the moreau envelope of nonsmooth convex functions over the fixed point set of certain quasi-nonexpansive mappings," Springer NY, pp. 345-390, 2011.

[23] Y. Censor and G. T. Herman, "On some optimization techniques in image reconstruction from projections," Applied $\mathrm{Nu}$ merical Mathematics, vol. 3, no. 5, pp. 365-391, 1987.

[24] I. Sezan and H. Stark, "Image restoration by the method of convex projections: Part 2-applications and numerical results," IEEE Transactions on Medical Imaging, vol. 1, pp. 95-101, 1982.

[25] Y. Censor and S. A. Zenios, "Proximal minimization algorithm withd-functions," Journal of Optimization Theory and Applications, vol. 73, pp. 451-464, 1992.

[26] A. Lent and H. Tuy, "An Iterative Method for the Extrapolation of Band-Limited Functions," Journal of Optimization Theory and Applications, vol. 83, pp. 554-565, 1981.

[27] Y. Censor, "Row-action methods for huge and sparse systems and their applications," SIAM review, vol. 23, pp. 444-466, 1981.

[28] Y. Censor, A. R De Pierro, and A. N. Iusem, "Optimization of burg's entropy over linear constraints," Applied Numerical Mathematics, vol. 7, no. 2, pp. 151-165, 1991.

[29] M. Rossi, A. M. Haimovich, and Y. C. Eldar, "Conditions for Target Recovery in Spatial Compressive Sensing for MIMO Radar," IEEE ICASSP, 2013.

[30] L.G. Gubin, B.T. Polyak, and E.V. Raik, "The Method of Projections for Finding the Common Point of Convex Sets," Computational Mathematics and Mathematical Physics, vol. 7, pp. $1-24,1967$.

[31] A. E. Çetin, O.N. Gerek, and Y. Yardimci, "Equiripple FIR Filter Design by the FFT Algorithm," IEEE Signal Processing Magazine, vol. 14, no. 2, pp. 60-64, 1997.

[32] A. Chambolle, "An algorithm for total variation minimization and applications," Journal of Mathematical Imaging and Vision, vol. 20, no. 1-2, pp. 89-97, Jan. 2004.

[33] R.G. Baraniuk, "Compressive sensing [lecture notes]," IEEE Signal Processing Magazine, vol. 24, pp. 118-121, 2007.

[34] P. L. Combettes and J.-Ch. Pesquet, "Proximal splitting methods in signal processing," Springer Optimization and Its Applications, pp. 185-212. Springer NY, 2011.

[35] Giovanni Chierchia, Nelly Pustelnik, Jean-Christophe Pesquet, and Béatrice Pesquet-Popescu, "Epigraphical projection and proximal tools for solving constrained convex optimization problems: Part i," CoRR, vol. abs/1210.5844, 2012.

[36] G. Chierchia, N. Pustelnik, J.-C. Pesquet, and B. PesquetPopescu, "An epigraphical convex optimization approach for multicomponent image restoration using non-local structure tensor," in IEEE ICASSP, 2013, 2013, pp. 1359-1363.

[37] M. Tofighi, K. Kose, and A. Enis Cetin, "Signal Reconstruction Framework Based On Projections Onto Epigraph Set Of A Convex Cost Function (PESC)," ArXiv e-prints, Feb. 2014.

[38] I. Johnstone B. Efron, T. Hastie and R. Tibshirani, "Least angle regression," Annals of Statistics, vol. 32, no. 2, pp. 407-499, 2004.

[39] F. Luisier, T. Blu, and M. Unser, "A new sure approach to image denoising: Interscale orthonormal wavelet thresholding," Image Processing, IEEE Transactions on, vol. 16, no. 3, pp. 593-606, March 2007.

[40] Zhou Wang, A.C. Bovik, H.R. Sheikh, and E.P. Simoncelli, "Image quality assessment: from error visibility to structural similarity," Image Processing, IEEE Transactions on, vol. 13, no. 4, pp. 600-612, April 2004.

[41] Kodak lossless true color image suite, "http://rOk.us/graphics/kodak/," 2013.

[42] A. Danielyan, V. Katkovnik, and K. Egiazarian, "Bm3d frames and variational image deblurring," Image Processing, IEEE Transactions on, vol. 21, no. 4, pp. 1715-1728, April 2012. 